\title{
COVID-19 creating challenges in a rehabilitation facility in Australia: A discussion paper
}

\author{
Janet Green*1, Joanne Vallance ${ }^{2}$, Rebekah Choong ${ }^{2}$, Georgina McBride ${ }^{2}$, Jeyanthi Arockiam², Sarah Jane Monaghan², \\ Sushma Prasad ${ }^{2}$, Linda K. Jones ${ }^{1}$ \\ ${ }^{1}$ College of Health and Medicine; School of Nursing, University of Tasmania, Australia \\ ${ }^{2}$ The Hills Private Hospital; Healthscope. Sydney, Australia
}

Received: November 17, 2020

DOI: $10.5430 /$ ijh.v7n1p44
Accepted: March 30, $2021 \quad$ Online Published: April 20, 2021

URL: https://doi.org/10.5430/ijh.v7n1p44

\begin{abstract}
The COVID-19 pandemic has brought about many challenges and alterations to long-standing procedures within rehabilitation facilities. These updates of the processes undertaken by staff in such settings have been radical and ongoing. COVID-19 impact on the healthcare system should never be underestimated nor under appreciated. The impact that this pandemic has had on rehabilitation care is unique. This has been the catalyst to identifying significant challenges and subsequent changes within rehabilitation and mental health practices. Social measures that have been implemented in the community have had exaggerated and magnified effects both on the physical and mental wellbeing of rehabilitation and mental health patients. Over the course of these adaptations and implementations, impacts on communication, mental health, workload and care efficiencies were identified and explored. This paper is unique because different disciplines have shared their experiences of the impacts of the COVID-19 pandemic and how they provide rehabilitation. The paper will focus on the communication challenges using masks, not being able to engage families and carers, restriction of group therapies, social isolation of patients and healthcare workers while adhering to guidelines set by NSW Health (Sydney, Australia).
\end{abstract}

Key Words: Rehabilitation, COVID-19, Challenges, Masks, Communication

\section{INTRODUCTION}

COVID-19 is a clinical syndrome caused by the coronavirus (SARS-CoV-2) and became a pandemic following an outbreak of viral pneumonitis, in 2019. It was first identified in Wuhan, Hubei, China. Acute respiratory distress syndrome (ARDS) and death are at the extreme end of the disease, however the disease manifests with a spectrum of symptoms ranging from mild upper respiratory tract infection to severe pneumonitis. It is now known that symptomatic people with coronavirus disease are the main disseminators, but asymptomatic people should not be underestimated. ${ }^{[1]}$
All areas of health care have been impacted by COVID-19, including rehabilitation services. Rehabilitation is required when a person is experiencing, or is likely to experience, limitations in their everyday functioning due to ageing or a health condition. ${ }^{[2]}$ Limitations in functioning can be due to injury, trauma and chronic diseases or disorders, and include difficulties in cognition, sight, hearing, communicating, ambulation, forming and sustaining relationships and/or keeping a job. ${ }^{[2]}$ Rehabilitation is about enabling individuals to return to their daily life activities, engage in fulfilling and meaningful life roles and maximise their wellbeing. ${ }^{[2]}$ When done

\footnotetext{
${ }^{*}$ Correspondence: Janet Green; Email: janet.green@utas.edu.au; Address: College of Health and Medicine; School of Nursing, University of Tasmania, Australia.
} 
properly, rehabilitation has a person-centred approach that can be delivered through a specialised rehabilitation program or integrated into other health programs and services. ${ }^{[2]}$

The purpose of this discussion paper is to outline the challenges faced by a multi-disciplinary team of health professionals and their rehabilitation patients during COVID-19. The rehabilitation patients are inpatients and outpatients of a private rehabilitation hospital located in Sydney, Australia.

\section{The hOSPITAL}

The "Hospital" is a medium sized sub-acute private health care facility providing rehabilitation and voluntary mental health care to the community in North West Sydney, NSW, Australia. The hospital has been the provider of these services for the past 11 years, previously it operated as an acute care facility, with those services transferred to a newly acquired site. The facility is structured with 92 rehabilitation and sub-acute medical beds and 19 mental health beds. Located on the site are four physiotherapy gymnasiums and a hydrotherapy pool.

Rehabilitation treatment of patients has an emphasis on continuity of care and specialises in post-operative orthopaedic, neurological and surgical rehabilitation. While the service and care provided for the mental health cohort of patients is centred on the treatment and recovery of mild mood disorders, anxiety, depression and drug and alcohol rehabilitation.

The impetus for this paper came when the first author, a Registered nurse and academic, became a rehabilitation patient in the hospital following a knee replacement. When this author arrived at the facility it became very obvious that a new normal had descended. Staff and patients wore masks, patients could not socialise or eat their meals together in the combined recreation and dining rooms. The companionship and camaraderie was missing. Visitors were allowed for only one hour per person per day, however unlike some other facilities, it could be a different person each day. Mask wearing was having an impact on the elderly and the hard of hearing. Not being able to see faces meant that a lot of vital expressive face information about moods was being missed.

The physiotherapy, exercise physiology and hydrotherapy spaces allowed only half the number of people allowed prior to COVID-19 which had implications for staffing.

\section{THE SCIENCE OF COVID-19}

Coronaviruses are a large family of viruses that have been around for many years, and they include the common cold and more recently COVID-19 (also known as SARS-CoV-2). Humans have built up an immunity to many of the coronaviruses, therefore the diseases are often not expressed as very severe in humans. ${ }^{[3]}$ Coronaviruses have been found in animals and these viruses can mutate and be passed onto humans, called zoonotic transfer. ${ }^{[3]}$ When zoonotic transfer occurs, the disease is severe because humans have no immunity to it, with SARS and MERS as examples. ${ }^{[3]}$ When a new strain of coronavirus is discovered it is referred to as a "novel" coronavirus. ${ }^{[3]}$

SARS-CoV-2 is a single-stranded RNA-enveloped virus. A key biological characteristic of SARS-CoV-2 is the presence of spike proteins that allow these viruses to penetrate host cells and cause infection. Glycosylated S proteins cover the surface of SARS-CoV-2 and bind to the host cell receptor facilitating viral cell entry. ${ }^{[4]}$ Viruses are not able to reproduce by themselves, therefore coronaviruses use their spikes to infiltrate living cells and get those cells to replicate the virus. $^{[4]}$

\section{IMMUNE SYSTEM OF THE ELDERLY}

The pathophysiology of the virus and how it affects the human body is now known. COVID-19 virus directly infects cells via the ACE2 Receptor. ${ }^{[5]}$ This is expressed in various organs, including the lung. The disease mechanism in severe cases is a "cytokine storm." This describes a cascade process whereby the virus leads to increased levels of cytokines that cause direct tissue damage, recruitment of neutrophils to tissues, and other pro-inflammatory effects. The damage can lead to acute respiratory distress syndrome (ARDS). ${ }^{[6]}$

Many of the COVID-19 deaths have occurred in the elderly with co-morbidities and health problems that might compromise their immune system. ${ }^{[7]}$ It became obvious early in the pandemic that those with pre-existing cardiovascular disease, hypertension and diabetes incur a disproportionately worse outcome. ${ }^{[8]}$ As the average age of patients/clients who utilise the rehabilitation services of the facility is 71.8 years of age, ${ }^{[9]}$ it would seem timely to outline the age-related changes to the immune system, and link them to the risk of COVID-19 infection.

Immuno-senescence is the name given to the natural, gradual deterioration of the immune system associated with age. Immuno-senescence involves both the host's ability to respond to infections and the development of long-term immune memory. ${ }^{[10]}$ The age-associated immune deficiency is a major factor in increased morbidity and mortality among the elderly. Immuno-senescence is not a random deterioration, rather it repeats an evolutionary pattern with most affected parameters under genetic control. It has been suggested that immune senescence is unavoidable because of the continuous, unavoidable exposure to viruses and bacteria. ${ }^{[10]}$

Age-dependent biological changes contribute to the onset 
of immune-senescence. With age, hematopoietic stem cells (HSC) diminish in their self-renewal capacity. There is a notable decline in the total number of phagocytes in older adults, with a decrease in their bactericidal activity. ${ }^{[10]}$ The cytotoxicity of natural killer (NK) cells is diminished. Cell-mediated immunity is decreased, making T-lymphocytes unable to modulate an adaptive immune response. Humoral immunity declines with a reduction in B-antibody cells. During ageing there is a decrease in the antibody function, response to vaccination and susceptibility to infections. ${ }^{[11]}$

A significant factor in the development of immunesenescence is the alterations in T-cell (thymus) development and function which increases susceptibility to both infectious and non-infectious diseases. The production of new naive lymphocytes and the functional competence of memory cells declines in the elderly leading to an increase in diseases such as cancer, chronic inflammatory disorders, and autoimmune disease. As the immune system wanes, infections in the elderly often present with non-specific signs and symptoms, thus providing problems in diagnosis and treatment. The beneficial effects of inflammation seen in younger people becomes detrimental later in life, resulting in changes in cytokine profile such as increased pro-inflammatory cytokines. ${ }^{[11]}$

It is noteworthy that lymphopenia, a marker of malnutrition has been found to be a negative prognostic factor in critically ill COVID-19 patients $^{[12]}$ with non-survivors of COVID-19 reported to have a significantly lower lymphocyte count than survivors. ${ }^{[13]}$

\section{NUTRITION TO SUPPORT THE AGING IM- MUNE SYSTEM DURING COVID-19}

Nutritional deficits are common in the elderly and are a contributing factor to weakening of the immune system. The ageing process is associated with enhanced catabolism and reduced anabolism, ${ }^{[14]}$ however nutritional support can improve the immune responses in older patients. ${ }^{[15]}$ Therefore, nutrition is an important consideration in rehabilitation of patients, and specifically in the elderly. An excellent publication by Bencivenga et al. ${ }^{[14]}$ has outlined how nutritional status can impact on the immune system of the elderly during COVID-19. There is a complex interplay between immunity and nutrition, with poor nutrition causing an impairment in cell-mediated immunity, cytokine production and decreased phagocytic function. ${ }^{[14]}$

A diet that has the right balance of vitamins and minerals will help to support the immune system of the elderly. Zinc enhances both the total number and function of T cells, with zinc also reported to increase natural killer cell (NK) activ- ity. ${ }^{[16]}$ Vitamin A has a role in modulating immune processes. It plays a role in epithelial and mucosal barrier generation, and contributes to immune cell homeostasis, differentiation and function. ${ }^{[17]}$ Furthermore, Vitamin E might be considered to be the most important nutritional component during COVID-19, because Vitamin E supplementation stimulates $\mathrm{T}$ cell function and confers some protection against upper respiratory tract infection in the elderly. ${ }^{[18]}$

Generally, Vitamin D deficiency is highly prevalent in the elderly worldwide. Vitamin D has been shown to modulate innate and adaptive immune system, and supplementation reduces viral replication and the production of proinflammatory cytokines. Furthermore, Vitamin D has a role in the differentiation of monocytes into macrophages and helps with the process of antigen presentation. Vitamin D is clearly very important for the elderly, and it has been proposed that high doses of vitamin $\mathrm{D}$ should be administered to COVID-19 patients. ${ }^{[19,20]}$

Vitamin $\mathrm{C}$ has a role in the regulation of $\mathrm{B}$ cell and $\mathrm{T}$ cell proliferation and differentiation, and they migrate to the sites of infection. In addition, Vitamin $\mathrm{C}$ promotes antimicrobial activity and antibody production, however it can be depleted during infections, especially in patients with the adult respiratory distress syndrome (ARDS) associated with COVID-19. Clinical trials investigating the efficacy of intravenous vitamin $\mathrm{C}$ in COVID-19 patients with ARDS are underway. ${ }^{[21]}$

The supplementation of probiotics could be beneficial for the elderly, with evidence that probiotics modulate the immune system. ${ }^{[22]}$ While the mechanisms by which probiotics exert their effects are partly unknown, it is known that probiotics are involved in modifying gut $\mathrm{pH}$, the production of antimicrobial compounds and stimulating immunomodulatory cells. ${ }^{[23]}$

\section{INFECTION CONTROL}

COVID-19 virus is transmitted between people through close contact and droplets. Airborne transmission may occur during aerosol generating procedures and support treatments. ${ }^{\text {[24] }}$ The WHO recommends airborne precautions for these procedures. For all, the most effective preventive measures include: maintaining physical distance (a minimum of 1 metre) from other individuals; performing hand hygiene frequently with an alcohol-based hand rub if available and if the hands are not visibly dirty, or with soap and water if hands are dirty ${ }^{[25]}$ Touching the mouth, nose and eyes is to be avoided and respiratory hygiene/ etiquette is practised by coughing or sneezing into a bent elbow or tissue, and then immediately disposing of the tissue; wearing a medical mask, originally with respiratory symptoms, however now currently recom- 
mended for the general public. ${ }^{[26]}$ The Centre for Disease and Prevention (CDC) ${ }^{[27]}$ suggest that the removal of masks should involve untying the strings and the outside corners of the mask should be handled by the ear loops. The front and rear of the facemask should not be touched during removal, and the outside corners of the mask should be put together. Care should be taken to avoid touching the eyes, mouth and nose when removing the mask. Hand hygiene should be performed after disposing of the mask. Routine cleaning and disinfection of environmental and other frequently touched surfaces is required. ${ }^{[27]}$ In the clinical setting the environmental cleaning has implications for staffing and organisational finances, because labour hours for cleaning high traffic areas have increased substantially.

\section{ORgANiSATIONAL ISSUES}

During the COVID-19 pandemic, the first challenge is the restriction of people in the same space, thereby impacting the delivery of care and service provision. Adhering to social distancing protocols is the overarching recommendation to prevent the spread of COVID-19. Staff must ensure such recommendations and protocols are adhered to for both themselves and patient safety. The number of people permissible on any site premises is determined by the "one person per 4 square metre rule." ${ }^{[28]}$ Social and physical distancing is now an essential practice in the prevention of micro-droplet exposure to virus transmission. By ensuring there is 4 square metres of space per person and maintaining a physical distance of at least 1.5 metres from others where possible, the likelihood of exposure to micro-droplets of others is reduced. ${ }^{[29]}$

The hospital has strictly maintained the recommended 1.5 metre distance rule during all patient interactions, with the exception of physical examination on patients. ${ }^{[28]}$ This has been achieved and is similar to any new practice or policy release, with education and communication methods. Despite this, social and physical distancing practices have grossly disrupted standard routines which have caused not only stress and anxiety to patients and staff, but fatigue and increased pressure on care providers in the hospital system.

It will not always be possible, however, for workers and others to maintain a 1.5 metre distance apart at all times at the workplace. For example, workers may have to work closely with each other or others because of the nature of the task and some tasks require workers to be in close proximity for the task to be carried out safely, for example checking scheduled drugs, and the physical care of patients.

\section{MASK WEARING}

Many of the challenges at the hospital have emerged as all health professionals and individuals who interact with pa- tients are now required to wear a face mask. The need for masks has presented challenges in communication, including the ability of staff and patients to read faces, as well as the impact of wearing masks for those who are cognitively impaired and/or hearing impaired. A recent publication by Green et al. ${ }^{[30]}$ has outlined the difficulties associated with wearing masks with infants. This work of Green et al. ${ }^{[30]}$ focusses on the impact on the growth and development of babies when they are trying to learn to read faces, however the paper reminds us that humans require visual facial cues when communicating.

There is a long history in some countries, especially in Asia, where wearing a mask has become a societal norm to safeguard from concerns such as pollution. ${ }^{[31]}$ Mask wearing has more recently presented certain struggles in the hospital setting with patient and staff communication and the establishment of trust and rapport building to engage in the delivery of care. While staff in the hospitals are particularly diligent and doing their part to slow the spread of coronavirus through the wearing face coverings, the masks often muffle voices and can make both verbal and physical communication challenging. This is particularly difficult for individuals with hearing and cognitive impairments. ${ }^{[30]}$

Humans generally rely on visual cues like facial expressions and lip reading to help understand what is being communicated verbally. The difference between a serious statement and a joke is often the smile on our faces or the tone of our voices. The COVID-19 pandemic has made this challenging since face masks are now recommended in hospital settings and these masks muffle our voices and hide our faces. ${ }^{[32]}$

\subsection{Implications}

Human beings are attuned to reading the facial expressions. Reading faces is a much-needed skill in our society and assists people gauge the emotions of others and regulate their behaviour and interactions. Humans have a coordinated package of communication cues including facial expressions, body language, hand gestures, words, pitch, tone, and face colour such as blushing. The communication cues act together to convey message and intent. ${ }^{[33]}$ Charles Darwin ${ }^{[34]}$ elucidated that reading facial expressions conferred an evolutionary advantage by aiding social interaction, reducing misunderstanding, and helping social groups function efficiently and harmoniously.

When a face mask is worn the eyes become a primary method of communication. Shakespeare is credited with saying "the eyes are the windows to your soul." "33] This infers that by looking into the eyes, mental states, emotions, desires, and intentions can be determined. The eyes and mouth are the most 
informative regions on the human face because they are the most expressive. The mouth region can express happiness with smiles, however for health professionals, concealing the mouth can be problematic when the staff want to appear friendly and approachable. ${ }^{[33]}$ Masks make it harder for the staff to determine emotions in their patients such as pain, discomfort, dismay and disdain. ${ }^{[34]}$ Masks can make users feel there is a physical barrier between themselves and the person they are trying to communicate with.

Approximately 5 percent of the world's population has disabling hearing loss ${ }^{[33]}$ and for people with hearing difficulties having the mouth covered is a major barrier. Masks can muffle speech, making hearing difficult. Those with hearing difficulties rely on visual cues and reading a person's lips and facial expressions. ${ }^{[35]}$ Clear plastic masks could be useful for those with hearing loss and those who care for them so they can continue to lip read.

\subsection{What is medical exemption}

While wearing a face masks remains challenging, at times, medical exemption for this may be necessary and appropriate. All exemptions in the healthcare environment should be on recommendation by a medical practitioner with discussion of appropriate alternatives and offer clear recommendations for risk-reducing measures. ${ }^{[36]}$ Inappropriate medical exemptions may inadvertently hasten viral spread and threaten public health. Medical exemption can be considered in:

(1) A person who is affected by a relevant medical condition, including problems with their breathing, a serious condition of the face, a disability or a mental health condition.

(2) Persons who are deaf or hard of hearing, where the ability to see the mouth is essential for communication.

\section{NuRSING CHALLENGES}

Nurses are the frontline healthcare professionals who have multiple roles and functions and are particularly important during this COVID-19 pandemic. These roles and functions involve providing education on infection prevention, implementing standard precautions, screening services, detection of infections signs and providing emotional support to those isolated because of COVID-19. ${ }^{[37,38]}$ In addition to providing safe care, nursing staff have to adapt to rapidly changing COVID-19 restriction requirements, providing psychological support to patients due to visiting restrictions. They are also required to spend time upskilling their clinical practice, attending training and attending to roles that were once undertaken by families and volunteers.

Clinical staffing shortages due to isolation leave and workplace fatigue, as well as initial insufficient personal protective equipment in the first COVID-19 wave (facial mask, gloves, impervious gowns, and eye protection) also contributed to fear and anxiety among nursing staff. Providing education to patients and families about infection control and spread and dealing with their own anxieties, are the primary stressors on nursing staff.

Continual investment and outlay are necessary to develop strong strategic recommendations and to integrate new knowledge, processes, policies and education. During outbreaks of new infectious diseases such as COVID-19 it is understandable that nurses will experience uncertainty, anxiety, and panic as the overall situation changes quickly, particularly when the disease was not yet considered under control. Supporting those nurses affected by disease, those under isolation, and healthcare staff is essential. Improving production and procurement of PPE is furthermore critical to ensuring security and safety and trust in the workplace.

\section{REHABILITATION MEDICAL STAFF}

The COVID-19 pandemic has been an unexpected shock to the rehabilitation teams because of interaction of the multidisciplinary team with the patient their family and community reintegration. The limits COVID-19 has placed on the patients and the teams have been enormous. Some patients avoid rehabilitation and instead decide to go home post operatively because of the fear of acquiring COVID-19 during the hospital stay. This has significantly limited their functional recovery post-operatively. The fear of acquiring the disease has made people more anxious and there is an exaggeration of the manifestations of anxiety and depression interfering with the outcomes and prolonging their stay. Limits placed on the visitors have also contributed to anxiety and restlessness in inpatients, especially in the non-English speaking patients. This is because they are unable to communicate with the other patients or staff, and in a few instances, this has led to early discharges. Furthermore, this has also manifested as increased perception of pain, insomnia and limited participation with therapists.

Children are not allowed in the facility, and this has emotionally deprived parents and grandparents of the comfort of their presence during the inpatient stay. A few patients have attempted self-harm or suffered an acute psychotic episode due to the exaggeration of their premorbid psychiatric illness, and isolation from their families and friends. The COVID19 restrictions have been challenging and have exaggerated these problems.

Interstate patients who have had to have unavoidable procedures during the pandemic have placed enormous stress on the patients and their families due to the separation and 
bureaucratic hurdles they face to visit, and for discharge planning. The willingness of patients taking up the required services post discharge have also declined due to the fear of acquiring infections from outsiders coming to provide care.

\section{Physiotherapy}

Respecting the 1.5 metre social distancing recommendation is not in the patient's best interests when doing so would compromise patient safety. Many of the patients are either presenting with a recent fall, or are at a high risk of a fall, and as a rehabilitation physiotherapist it is essential to stay within an arm's length of these patients when they are doing certain standing exercises that may challenge their balance. There are many patients that are dependent on physical assistance or supervision for mobility and transfers and so, need to be physically touched. In the hospital most sessions are conducted in the gymnasiums where several people are exercising side by side. COVID-19 has resulted in a cap on the number of people that can be in the gymnasium simultaneously and requires that patients are carefully scheduled. However, it can mean that it takes longer to provide physiotherapist to all of the rehabilitation patients.

\section{OCCUPATIONAL THERAPY}

The role of occupational therapy is varied in a rehabilitation setting as rehabilitation is a complex process. ${ }^{[39]}$ All therapies provided however have a common goal: to restore function and maximize a patient's independence in their everyday activities while promoting a good quality of life. The demographics of patients who are treated in an inpatient rehabilitation unit are typically over 65 years of age and have multiple comorbidities, commonly but not limited to respiratory, cardiac, neurological or oncologic. ${ }^{[40]}$ Typically, this rehabilitation population requires a high level of physical contact when delivering therapies, for example, transfer practice, mobility assistance and personal care assessments and retraining. ${ }^{[40]}$ With the new COVID-19 restrictions a vast majority of occupational therapy procedures and treatments in a rehabilitation setting have had to be changed accordingly.

Wearing masks while providing therapy means that communication with patients has been impacted significantly. ${ }^{[41]} \mathrm{A}$ significant portion of the patients experience hearing impairments. The use of masks not only muffles the voice making it harder for patients to distinguish what is being said, but also prevents patients from reading lips or interpreting what is being said via body language. ${ }^{[42]}$ Furthermore, the social distancing restrictions hinder therapists from sitting in closer proximity to patients to assist with verbal communication. This has not only lengthened the time of consultations (due to repetition of questions being asked) but has also impacted on rapport being built with patients. Camaraderie with patients has been impacted upon as patients get frustrated with not being able to hear questions properly, as well as struggle to realise when therapists are laughing with them or even just sharing a smile.

Cognitive assessments which often include repetition of verbal sentences and following a set of verbal instructions/commands have also been impacted upon due to the communication difficulties. Scores gained by these standardized and non-standardized cognitive assessments may therefore not be as accurate or be a correct reflection of a patient's cognitive abilities due to these difficulties with hearing. ${ }^{[43]}$

Treatments which involve common equipment in a confined space are not recommended. Therefore, educating and practicing car transfers to abide by specific orthopaedic precautions (for example, posterior hip replacement precautions) is no longer conducted. Instead, therapists have had to adapt and educate their patients individually in the patient's room, modelling the procedure with furniture available in the room. This means patients are not provided with a physical practice and instead have to translate the procedure taught in their room to the real-life scenario.

Group therapies such as falls prevention education, upper limb therapy and breakfast groups have been ceased. Occupational therapists previously provided a "breakfast group" every Wednesday and Friday. This group enabled therapists to perform kitchen assessments (that is, assessing a patient's functional ability in preparing a meal and drink for themselves) while also providing patients with an enjoyable social environment. Instead, therapists must now provide one on one assessments with patients, thus increasing the amount of time therapists require to complete assessments. Ceasing this breakfast group also prevents a therapist from assessing a patient's social interactions, attention and cognition in a group setting.

Home visits in which an occupational therapist takes patients back to their residence to assess their safety and function in their home environment, are no longer completed due to COVID-19 restrictions. This impacts on patients discharge destinations as they no longer have the opportunity to demonstrate to therapists their abilities in their own environments. Access visits in which a therapist goes out to assess a patient's home without the patient being present have also been restricted due to COVID-19. These visits are now only allowed to be performed with special exemption from the hospital general manager. Therefore, therapists have had to adapt to how they can assess a patient's home. Therapists are now having to rely on patient's families and friends to 
provide photos, verbal explanation and measurements of the home environments. Difficulties with using these methods include: family may not be able to visit or provide photos in a timely manner, measurements of furniture heights, doorway clearances and so forth may not be accurate, equipment is often recommended over permanent modifications (for example, over toilet aids prescribed in lieu of grab rail instalment) and equipment cannot be trialled in the home environment to ensure it is suitable.

There are limitations to the involvement of family members during the rehabilitation process due to restricted visiting hours and one visitor per patient. Family and friends play an important role in a patient's rehabilitation journey; they not only provide emotional support but can also assist with continuation of therapy. Therapists often try to engage family members in assisting with delivering therapy to patients outside of the scheduled therapy times. By doing this, patients benefit from a continuity of practice on the ward, as well as involving the family in carer training in anticipation for the patient's discharge. For example, in patients recovering from stroke, family and friends are often taught how to assist with upper limb therapy (for example, applying functional electrical stimulators, passive range of motion stretches, increased repetitions of exercises outside of scheduled therapy times), cognitive therapy, functional retraining (for example, toileting, cutting up meals). Due to the limited visitation of one hour, the ability to engage families in delivering continued therapy is surpassed by family using the time to socialize and provide emotional support. Thus, patients receive a less amount of overall therapy during each day which subsequently could lead to a lengthened inpatient admission.

Patients are no longer allowed to leave the hospital premises (gate leave) unless it is for necessary appointments. Pre COVID-19, patients were allowed to attend social outings in the community and go home for a few hours once it was deemed safe by the treating multidisciplinary team. It is during these outings that patients could assess how they managed in their home environment, car transfers and their mobility and transfers within the community. If difficulties occurred during this leave period, patients and their family/friends were able to inform the therapists and treatments to overcome these issues could commence (for example, requiring further equipment recommendations, or further practice of transfers).

The length of time to complete occupational therapy interventions has increased due to additional COVID-19 precautions (for example, donning/doffing PPE, no group therapies, additional risk assessments and checklists) has increased and consequently has impacted on the amount of active therapy time/service delivery in a working day.

Moving forward, occupational therapists delivering services in an inpatient rehabilitation unit during COVID-19 or other pandemics in the future, should consider the following recommendations:

(1) Provide additional time to complete assessments and interventions. Therapists should try to allocate additional time in their day to take into account completing new COVID-19 checklists/risk assessments before some therapies, donning/doffing of PPE between patients and extra time for communicating with patients.

(2) For communicating with patients while wearing masks, therapists should make conscious efforts to speak slowly, enunciate words and reduce background noise when conversing with patients, to provide them with the best opportunity to hear you. ${ }^{[4]}$

(3) Be aware that cognitive assessments which use repetition and following verbal commands; such as the Standardized Mini-Mental Status Examination Functional, ${ }^{[45]}$ Montreal Cognitive Assessment ${ }^{[46]}$ and Addenbrooke's Cognitive Examination, ${ }^{[47]}$ scores may be affected. These assessments may not be as accurate in screening and assessing cognition due to mask use and abiding by social distance procedures. Therapists should use these assessments in conjunction with functional assessments (for example, kitchen assessments, medication sorting) to assess cognition.

(4) Provide a worksheet which demonstrates how to take accurate measurements of furniture, stair dimensions and door clearances. This worksheet assists family and friends to know what measurements are required and how to take photographs of these items at appropriate angles. This allows therapists to gain knowledge of the patient's home environment without performing an access home visit; assisting in equipment prescription, falls prevention education and transfer retraining (from heights of patient's furniture).

(5) Updating a patient's next of kin on their current functional status frequently through phone call or email correspondence. This allows the patient's family and/or friends to still be involved with the patient's rehabilitation journey as well as providing them with an opportunity to highlight any other concerns they may have for the patient's discharge plan.

\section{HYDROTHERAPY}

Reduced capacity in the hydrotherapy pool by approximately $50 \%$ has led to the scheduling of extra classes which can be 
problematic for staffing. All staff and patients are wearing masks in the pool. There are the variations in attitudes to masks, and those people who are bothered or concerned by the masks do not tend to interact and converse in the pool. In the pool the communication difficulties can be heightened in those who use facial expressions or lip reading to communicate (for example, those with dementia or deafness). Some people, especially those with shortness of breath, anxiety or claustrophobia find masks oppressive. It is also acknowledged that having to wear a mask in the hydrotherapy pool would be a very strange experience.

\section{Dietitian}

From the $25^{t h}$ of March through to 21 April 2020 all elective surgery in NSW was suspended ${ }^{[28]}$ in an attempt to minimise PPE use. It was during this time that the hospital saw a significant shift in patient type from predominately surgical recovery to more medically unwell, and a much weightier list of co-morbidities. Although the hospital population dropped significantly during this time, the number of dietitian referrals remained constant, yet more complex. This was due to the presenting health conditions and comorbidities, and meant that patients had increased nutritional needs, yet tended to have decreased appetites. The number of special diets ordered was significantly increased, increasing the workload on catering, despite their having a reduced number of staff reflective of our patient load.

The enforcement of social distancing and wearing of PPE, specifically masks, had made communication with patients very difficult. The additional distance of $1.5 \mathrm{~m}$ between staff and patient has led to increased volume of speaking, consequently increasing background noise for all patients and so reducing the effectiveness of hearing aids and other hearing devices.

When asking and trying to demonstrate a request for patient to show their teeth and tongue, open their mouth and so forth; this is often missed by patients and this part of the assessment has occasionally had to be omitted or significantly abbreviated.

Future recommendations for dietitians working within COVID-19 restrictions and the need for PPE may include the use of face shields rather than masks, so that patients can still lip-read and have a better understanding of facial expressions, demonstrations and cues.

\section{MENTAL HEALTH ISSUES}

The COVID-19 pandemic has an influence on clients with mental health issues, and mental health services are inevitably affected by it. Inpatient therapy is mainly focused on recovery models, consumer centred care and trauma informed care. The inpatient programme for mental health consumers is a 21-day programme which provides a combination of psychotherapy, counselling, medication management and exercise including hydrotherapy pool and regular consultation with psychiatrists. Nurses in mental health settings have greater responsibility to adhere to the current COVID guidelines.

Wearing face masks in all areas, apart from when clients are in the room by themselves is essential and strongly recommended. Nurses, therefore, need to provide masks to the inpatients and also need to be remind them that they must be changed in the recommended time frame. Reminding clients to wear a face mask is a challenge for nurses as clients may not be able to understand the requirement due to the nature of their illness. It has been noted at times that clients are overly concerned with the wearing of masks, and should therefore be made aware of their necessity in order to prevent infection. Nurses' assessments require an understanding of clients' facial expressions and similarly clients should understand nurses' facial expressions. The wearing of a mask can limit facial clues thereby hindering the therapeutic relationship. Clients, when attending inpatient therapy must also wear masks. However, the wearing of a mask may have a negative influence on the therapy session as it prevents communication with facial expressions.

Social distancing in the hospital is recommended. One of the challenges in maintaining social distance is the smoking area. There is a tendency for clients to gather and spend more time in the smoking area. Even though clients are reminded daily of the smoking policies, enforcing adherence to the policy is a challenge for nurses. Nicotine replacement therapy is highly recommended for clients.

Visiting restrictions also play a vital part in recovery as clients are not able to see their loved ones. Normally clients are able to understand the importance of visiting restrictions and leave restrictions. These restrictions have impacted on their social interactions which result in more anxiety and depressive moods, thereby requiring medication to manage their level of stress. It is advised that in order to reduce loneliness and maintain connection between clients and their families that they communicate through online chatting or video calls. Clients are advised to cancel their outside appointments while they are inpatients, or be advised to use Zoom ${ }^{\circledR}$ for their appointments as a way of preventing unnecessary exposure to the community.

Due to social distancing and the number of people allowed in the group room and dining room, the therapy is divided into two groups and meal-times are also set for two sittings. 
Clients are able to understand the reasons for dividing these sessions. Some reflected upon the small group sessions providing more interaction. Meal-time may be challenging for some as they prefer to sit with their preferred peers. Hydrotherapy provides many positive outcomes for mental health client's symptom management. Cancellation of hydrotherapy also impacted upon exercise therapy and inpatient programme structure.

Nursing care requires more counselling support for clients, especially during weekends, as there are limited group activities. Nurses need to encourage practicing cognitive behavioural therapy and mindfulness rather than relying on medications for symptoms management, being secondary to COIVD restrictions. It is noted that due to COVID restrictions and limited exercise opportunities, clients have a tendency to consume unhealthy diets which also relates to utilising more medications for their symptom management. It is evident that when carrying out a weekly weighing in on Sundays, as part of the metabolic monitoring policy, that the majority of clients gain weight compared to their initial weight at the time of assessment. Nurses have the responsibility to address healthy eating education as part of their care plan.

\section{WHO RECOMMENDATIONS}

Many people, particularly older adults, with long-term care needs have been isolated in homes or facilities for many weeks, leading to reduced social contact and disrupted and changed routines. Individuals with long-term care needs, such as people living with dementia, experience changes in both their physical and cognitive status. Rapid changes to their routine may also increase their vulnerability to preexisting conditions.

Specifically, during extended stay, patients have struggled with the restrictions to socialize with fellow patients or to receive visits from their family and friends. Families have also displayed distress that they are not able to visit and oversee the care provision and recovery.

Long-term care facilities have recognized the importance of supporting patients with social contacts and have introduced innovative solutions, such as technical tools that enable virtual contact with their families and friends. ${ }^{[25]}$ Staff have facilitated contact with family and friends by phone, the online instant messaging or written messages if access is restricted.

One of the first measures adopted in almost all countries has been restricting visitors to long-term care facilities. While this measure is relatively easy to implement, it is increasingly recognized that it has an enormous impact on the well-being of both patients of long-term care facilities and their families, and that, particularly where the patient has a cognitive impairment, a lack of understanding of why the visits have stopped may generate additional distress. Concerns have also been expressed that many family members provide care in their regular (sometimes daily) visits, and that not allowing them to visit at a time when the staff of long-term care facilities may already be overburdened due to increased complexity of care and lower staffing ratios may compound staffing problems. ${ }^{[25]}$

While the COVID-19 pandemic has brought public attention to the immediate impacts of the pandemic, and the need to be prepared for present contingencies and possible future waves, it has also shown that there are major structural challenges that need to be addressed to improve the safety and resilience of patients and staff in the healthcare environment.

\section{Conclusion}

While physical distancing is helping to prevent people from contracting the coronavirus, it has come with detrimental effects, including isolation, loneliness, anxiety, problems with psychological well-being and financial burden. The multidisciplinary team have worked tirelessly during the COVID19 epidemic to provide a quality rehabilitation experience for the clients in the facility. While doing so, they recognise it is important for the hospital to balance safeguarding the health of staff, patients and families with implementing policies and strategies to decrease conflicts stemming from disease-related discrimination, stigmatization and isolation. The family is integral for successful rehabilitation, so it is important for organizations to establish clear visiting policies that provide balance between the safety measures that decrease COVID-19 transmission and the need for people to maintain their psychological well-being.

\section{CONFLiCTS OF INTEREST Disclosure}

The authors declare no conflicts of interest. 


\section{REFERENCES}

[1] Heneghan C, Brassey J, Jefferson T. COVID-19: What proportion are asymptomatic? April 6, 2020. Available from: https://www.cebm.net/covid-19/covid-19-what-p roportion-are-asymptomatic/

[2] World Health Organisation. nd. Rehabilitation. Available from: https ://www . who.int/news-room/fact-sheets/detail/r ehabilitation

[3] Liu YC, Kuo RL, Shih SR. COVID-19: The first documented coronavirus pandemic in history. Biomedical Journal. 2020 Aug 1; 43(4): 328-33. PMid:32387617. https://doi .org/10.1016/j . bj . 202 0.04 .007

[4] Huang Y, Yang C, Xu XF, et al. Structural and functional properties of SARS-CoV-2 spike protein: potential antivirus drug development for COVID-19. Acta Pharmacologica Sinica. 2020 Sep; 41(9): 11419. PMid:32747721. https://doi .org/10.1038/s41401-020-0 485-4

[5] Sriram K, Insel E, Loomba R. What is the ACE2 receptor, how is it connected to coronavirus and why might it be key to treating COVID-19? The experts explain. The Conversation. 2020. Available from: https://theconversation.com/what-is-the-ace 2-receptor-how-is-it-connected-to-coronavirus-and -why-might-it-be-key-to-treating-covid-19-the-exp erts-explain-136928

[6] Mehta P, McAuley DF, Brown M, et al. COVID-19: consider cytokine storm syndromes and immunosuppression. The Lancet. 2020 Mar; S0140673620306280. https : //doi .org/10.1016/S0 140-6736 (20) 30628-0

[7] Abdulamir AS, Hafidh RR. The Possible Immunological Pathways for the Variable Immunopathogenesis of COVID-19 Infections among Healthy Adults, Elderly and Children. Electronic Journal of General Medicine. 2020; 17(4): em202. https://doi.org/10 .29333/ ejgm/7850

[8] Liu PP, Blet A, Smyth D, et al. The science underlying COVID-19: implications for the cardiovascular system. Circulation. 2020; 142(1): 68-78. PMid:32293910. https://doi.org/10.1161/CIRCULAT IONAHA .120 .047549

[9] Australian Rehabilitation Outcomes Centre. University of Wollongong, Australia. 2019. Available from: https://www .uow.edu.au /ahsri/aroc/

[10] Gruver AL, Hudson LL, Sempowski GD. Immunosenescence of ageing. The Journal of Pathology: A Journal of the Pathological Society of Great Britain and Ireland. 2017; 211(2): 144-156. PMid:17200946. https://doi.org/10.1002/path.2104

[11] Alam I, Almajwal AM, Alam W, et al. The immune-nutrition interplay in aging-facts and controversies. Nutrition and Healthy Aging. 2019; 5(2): 73-95. https://doi.org/10.3233/NHA-170034

[12] Wu C, Chen X, Cai Y, et al. Risk Factors Associated with Acute Respiratory Distress Syndrome and Death in Patients with Coronavirus Disease 2019 Pneumonia in Wuhan, China. JAMA Intern Med. 2020; 180(7): 934-943. PMid:32167524. https://doi.org/10.1001/ jamainternmed.2020.0994

[13] Huang I, Pranata R. Lymphopenia in severe coronavirus disease2019 (COVID-19): systematic review and meta-analysis. Journal of Intensive Care. 2020; 8(1): 1-10. PMid:32483488. https : //doi.org/10.1186/s40560-020-00453-4

[14] Bencivenga L, Rengo G, Varricchi G. Elderly at time of CoronaVirus disease 2019 (COVID-19): possible role of immunosenescence and malnutrition. GeroScience. 2020; 42: 1089-1092. PMid:32578073. https://doi.org/10.1007/s11357-020-00218-9

[15] Pansarasa O, Pistono C, Davin A, et al. Altered immune system in frailty: genetics and diet may influence inflammation. Ageing
Research Reviews. 2019; 54: 100935. PMid:31326616. https: //doi.org/10.1016/j.arr.2019.100935

[16] Barnett JB, Dao MC, Hamer DH, et al. Effect of zinc supplementation on serum zinc concentration and $\mathrm{T}$ cell proliferation in nursing home elderly: a randomized, double-blind, placebo-controlled trial The American Journal of Clinical Nutrition. 2016; 103(3): 942-951. PMid:26817502. https://doi.org/10.3945/ajcn.115.11518 8

[17] Huang Z, Liu Y, Qi G, et al. Role of vitamin A in the immune system. Journal of Clinical Medicine. 2016; 7(9): 258. PMid:30200565. https://doi.org/10.3390/jcm7090258

[18] Lee GY, Han SN. The role of vitamin E in immunity. Nutrients. 2018; 10(11): 1614. PMid:30388871. https://doi.org/10.3390/nu 10111614

[19] Jakovac H. COVID-19 and vitamin D-Is there a link and an opportunity for intervention? American Journal of PhysiologyEndocrinology and Metabolism. 2020; 318(5): E589-E589. PMid:32297519. https://doi.org/10.1152/ajpendo.00138. 2020

[20] Grant WB, Lahore H, McDonnell SL, et al. Evidence that vitamin D supplementation could reduce risk of influenza and COVID-19 infections and deaths. Nutrients. 2020; 12(4): 988. PMid:32252338. https://doi.org/10.3390/nu12040988

[21] Carr AC. A new clinical trial to test high-dose vitamin $\mathrm{C}$ in patients with COVID-19. Critical Care. 2020; 24(1): 1-2. PMid:32264963. https://doi .org/10.1186/s13054-020-02851-4

[22] Pae M, Meydani SN, Wu D. The role of nutrition in enhancing immunity in aging. Aging and Disease. 2012; 3(1): 91.

[23] Amara AA, Shibl A. Role of Probiotics in health improvement, infection control and disease treatment and management. Saudi Pharmaceutical Journal. 2015; 23(2): 107-114. PMid:25972729. https://doi.org/10.1016/j.jsps.2013.07.001

[24] Tran K, Cimon K, Severn M, et al. Aerosol generating procedures and risk of transmission of acute respiratory infections to healthcare workers: a systematic review. PloS one. 2012; 7(4). PMid:22563403. https://doi.org/10.1371/journal.pone.0035797

[25] World Health Organisation. Infection prevention and control during health care when novel coronavirus (nCoV) infection is suspected. Interim Guidance. Geneva: World Health Organization; 2020. Available from: https://www. who.int/publications/i/item/10 665-331495

[26] World Health Organisation. Rational use of personal protective equipment (PPE) for coronavirus disease (COVID-19) - Interim guidance (19 March 2020). Available from: https://relief web. in t/report/world/rational-use-personal-protective-e quipment-ppe-coronavirus-disease-covid-19-interim

[27] Centers for Disease Control and Prevention. 2020b. How to Wear Masks. Updated Aug. 7, 2020. Available from: https: //www.cdc.gov/coronavirus/2019-ncov/prevent-getti ng-sick/how-to-wear-cloth-face-coverings .html

[28] NSW Health. Four square metre rule. Sydney, Australia: NSW Health; 2020 [Cited 2020 Oct]. Available from: https://www.nsw. gov.au/covid-19/what-you-can-and-cant-do-under-rul es/four-square-metre-rule\#maximum-number-of-people

[29] Safe Work Australia. COVID-19 Information for Workplaces: Physical distancing. Canberra, Australia. SWA; 2020 [Cited 2020 Sept]. Available from: https://www.safeworkaustralia.gov . au/covid-19-information-workplaces/industry-infor mation/office/physical-distancing

[30] Green J, Petty J, Staff L, et al. The implications of face masks for babies and families during the COVID-19 pandemic: A dis- 
cussion paper. Journal of Neonatal Nursing. 2020. PMid:33162776. https://doi.org/10.1016/j.jnn.2020.10.005

[31] Wong B. Why east Asians were wearing masks long before COVID19. HuffPost. 19 September 2020. Available from: https : //rb.g y/qq2aeh

[32] Watt D. The science of how you sound when you talk through a face mask. The Conversation. 2 July 2020. Available from: https://theconversation.com/the-science-of-how-you -sound-when-you-talk-through-a-face-mask-139817

[33] Ong S. How face masks affect our communication. BBC Future. 9 June. 2020. Available from: https: //www.bbc.com/future/article/20200609-how-fac e-masks-affect-our-communication

[34] Darwin C. The expression of the emotions in man and animals. USA: Oxford University Press; 1872. PMid:28995415. https: //doi.org/10.1037/10001-000

[35] Fortin J. Masks Keep Us Safe. They Also Hide Our Smiles. The New York Times. 23 June. 2020. Available from: https://www . nytimes.com/2020/06/23/style/face-m ask-emotion-coronavirus.html

[36] Dorfman D, Raz M. Mask Exemptions During the COVID-19 Pandemic-A New Frontier for Clinicians. JAMA Health Forum. 2020; July 10. https://doi.org/10.1001/jamahealthforum. 2020 .0810

[37] Jernigan DB. Update: public health response to the coronavirus disease 2019 outbreak-United States, February 24, 2020. MMWR. Morbidity and mortality weekly report. 2020; 69: 216-219. PMid:32106216. https ://doi.org/10.15585/mmwr.mm6908e1

[38] Patel A, Jernigan DB. 2019-nCoV CDC Response Team. Initial public health response and interim clinical guidance for the 2019 novel coronavirus outbreak - United States, December 31 2019-February 4 2020. Morbidity and Mortality Weekly Report. 2020; 69(5): 140-146 PMid:32027631. https ://doi.org/10.15585/mmwr.mm6905e1
[39] Wade DT. Applying the WHO ICF framework to the rehabilitation of patients with cognitive deficits. In Halligan PW and Wade DT Edit. 2005 Effectiveness of Rehabilitation for Cognitive Deficits. Oxford: Oxford Press; https://doi.org/10.1093/acprof : oso/ 9780198526544.003 .0004

[40] McNeary L, Maltser S, Verduzco-Gutierrez M. Navigating coronavirus disease 2019 (Covid-19) in physiatry: A CAN report for inpatient rehabilitation facilities. PM\&R. 2020; 12(5): 512-515. PMid:32196983. https ://doi.org/10.1002/pmrj.12369

[41] Chodosh J, Freedman ML, Weinstein BE, et al. Face masks can be devastating for people with hearing loss. BMJ. 2020; 370. PMid:32646862. https ://doi .org/10.1136/bmj .m2683

[42] York D. How to build rapport ... while wearing a mask. 28 September 2020. Available from: https://hbr.org/2020/09/how-to-bui ld-rapport-while-wearing-a-mask

[43] Parada JC, Hillyer J, Parbery-Clark A. Performance on the standard and hearing-impaired Montreal Cognitive Assessment in cochlear implant users. International Journal of Geriatric Psychiatry. 2020 Apr; 35(4): 338-47. PMid:31989675. https ://doi.org/10.100 2/gps. 5267

[44] Mendel LL, Gardino JA, Atcherson SR. Speech understanding using surgical masks: a problem in health care?. Journal of the American Academy of Audiology. 2008 Oct 1; 19(9): 686-95. PMid:19418708. https://doi.org/10.3766/jaaa.19.9.4

[45] Rovner BW, Folstein MF. Mini-mental state exam in clinical practice. Hosp Pract (Off Ed). 1987; 22(1A): 99, 103, 106, 110. PMID: 3100557.

[46] Nasreddine ZS, Phillips NA, Bedirian V, et al. The Montreal Cognitive Assessment, MoCA: a brief screening tool for mild cognitive impairment. Journal of the American Geriatric Society. 2005; 53(4): 695-699. PMid:15817019. https://doi.org/10.1111/j.1532 $-5415.2005 .53221 . \mathrm{x}$

[47] Larner AJ. Addenbrooke's Cognitive Examination-revised (ACE-R) in day-to-day clinical practice. Age and Aging. 2007; 36(6): 685-686. PMid:17881421. https://doi.org/10.1093/ageing/afm112 